\title{
Automatic fibrosis quantification by using a k-NN classificator
}

\author{
E.Romero, J.M. Raymackers, B. Macq and O. Cuisenaire
}

\begin{abstract}
This work presents an automatic algorithm to measure fibrosis in muscle sections of $m d x$ mice, a mutant species used as a model of the Duchenne dystrophy. The algorithm described herein automatically segments three different tissues: Muscle cell tissue (MT), Pure collagen fiber deposit (CD) and cellular infiltrates surrounded by loose collagen deposit (CI), by using a statistical classifier based on the $k$-Nearest Neighbour ( $k$-NN) decision rule in the RGB color space. The algorithm is trained by selecting a number of correctly classified pixels from each class. The $k$-NN rule classifies other pixels in the class that is most represented among the $k$ nearest training samples in the RGB space, which is efficiently implemented with a fast k-distance transform algorithm. All extracted areas are quantified in absolute $\left(\mu \mathrm{m}^{2}\right)$ and relative (\%) values. For validation of this method, the different tissues were manually segmented and their quantifications statistically compared with those obtained automatically. Statistical analysis showed interoperator variability in manual segmentation. Automatic quantifications of the same areas did not differ significantly from their mean manual evaluations. In conclusion, this method produce fast, reliable and reproducible results.
\end{abstract}

Keywords - Automatic morphometry, fibrosis morphometry, muscle measurements, $k$-NN.

\section{INTRODUCTION}

Fibrosis is a common consequence of a large number of pathologies in different organs and its quantification stands for a fundamental step towards correct evaluations. These kind of measurements have been addressed by different methods. Semiquantitave stereological approaches have been used in histomorphometrical problems whose aim was to quantify interstitial fibrosis by using the point-counting technique [1], [2], [3], [4]. However, provided that in muscular distrophies, the fibrotic areas are not homogeneously distributed [5], [6], [7], [8], [9], the amount of work needed for an operator to obtain representative measurements of each of the different pathologic events becomes a considerable burden. In addition, manual measurements are highly time consuming, not extremely precise and depend on the skill of the experimenter; resulting in a large both, inter and intra-observer variabilities. On the contrary, digital image analysis provides more reliable and reproducible quantitative measurements than conventional methods [10].

The present investigation presents the development and validation of a new automatic method to quantify the interstitial fibrosis in muscle images. Firstly, a few samples of correctly classified pixels are manually selected to obtain

Eduardo Romero and Benoît Macq are with the Communications and Remote sensing laboratory and Jean Marc Raymackers with the Department of physiology and pharmacology, all at Université Catholique de Louvain, Belgium. Olivier Cuisenaire is with the Signal Processing Laboratory, EPFL, Swiss Federal Institute of Technology. E-mail: romero@tele.ucl.ac.be. a good representation of the different tissues (categories) in the image to classify. Next, these picked pixels are used to train a statistical classifier in the RGB color space. The classifier used here is based on the $k$-Nearest Neighbors $(k$ NN) classification rule [11], [12], which is a technique for non-parametric pattern classification. This rule classifies a given pixel within the category most heavily represented amongst its $k$-Nearest Neighbors, in the pattern space. Every pixel in the RGB space is then associated to a determined category, according to the $k$-NN rule. Finally, the complete data set with the images of the whole muscle section is then automatically classified by using an efficient implementation of this $k$-NN rule.

\section{Materials And Methods}

\section{A. Histological Material}

Male $m d x$ mice used in an unrelated experiment were anaesthetised by a subcutaneous injection of a mixture of ketamine hydrochloride $50 \mathrm{mg} / \mathrm{ml}$ (ketalar) and xylazin hydrochloride $2 \%$ (Rompun). The tibialis anterior muscle was then dissected and completely sectioned. Serial $7 \mu m$-thick transverse sections were made from the mid-belly region of the muscles, using a cryostat (Reichter-Jung Cryocut E), and mounted on microscope slides (Superfrost). Then, sections were stained with $1 \%$ picro-Sirius red (Direct red 80, Fluka Sigma), washed with two changes of acetic acid water, dehydrated and mounted with a resinous medium. Sirius red stains CD in a deep red color and MT in yellow, while CI areas are stained in a pink difuse color (see Figure $1)$.

\section{B. Image Acquisition}

Images were acquired with a Fujix HC-2000 $(1280 \times 1024$ pixels to $2560 \times 2048$ ) high resolution digital camera coupled to an Olympus BX 50 microscope with an Olympus U-BMAD and U-TU1 X adapters (Olympus, Optical company, Ltd., Japan). Microscope plate was remote controlled with a step by step controller PRIOR H-101, which was communicating to a Dell PC Penthium III, $300 \mathrm{M} \mathrm{Hz}$ with 384 RAM $M b$ through a $R S 232$ line. Analog images were then digitized at 24 bits in different formats: TIFF, JPEG and BMP by using Photograph II SH-WSII software. Control of the automatic microscope plate and Photograph II software was performed by a home made software written in Winbatch 2000 (WindowWare Inc) under Windows NT4.

Use of intermediate lens and $\mathrm{a} \times 20$ power objective yielded a total magnification of $\times 900$. Screen image size 
was $199 \times 152 \mu \mathrm{m}^{2}$ for a $1280 \times 1000$ pixel image, resulting in a total resolution of $0.011 \mu \mathrm{m}^{2} /$ pixel.

\section{Image Analysis}

A transversal section of muscle was digitized and one image was randomly selected to perform manual-automatic comparisons. Segmented image surfaces of MT, CD and CI surfaces were quantified in both raw pixels and $\mu m^{2}$.

\section{C.1 The Bayes risk and k-NN decison approach}

The basic structure of a general decision problem consists of a set of decisions $D$, a parameter space $\Omega$, a probability distribution function $p(\boldsymbol{\Omega})$ and a utility function $u(\delta(\omega))$, whose maximum is the best decision $\delta^{*}(w)$. Decision rules are evaluated in terms of their average loss function $l(\delta(\mathbf{x}), \omega)$ with respect to the data which might arise and is given by

$$
l(d w)=\sup _{d_{i} \in D}\left\{u\left(d_{i}(\omega)\right)-u(d(\omega))\right\}
$$

where $\mathbf{x}$ is the observation and $\omega$ a particular event. The risk function $r(\delta, \omega)$ of a decision rule $\delta$ can then be defined as

$$
r(\delta, \omega)=\int l(\delta(\mathbf{x}), \omega) \mathbf{p}(\mathbf{x} / \omega) \mathbf{d} \mathbf{x}
$$

Classical decision theory focuses on the decision rule which minimises expected risk (or so-called Bayes risk)

Classification problems can always be placed between these two situations: either there is a complete knowledge of the statistical properties of the problem or the unique statistical knowledge is that which can be inferred from some samples. In the former case, a standard Bayes analysis will yield an optimal choice with a corresponding minimum probability of error of classification: the Bayes risk $R^{*}$. In the other case, the decision to assign the observation $\mathbf{x}$ into a given category $\theta$ is dependent on the correct classfication of $n$ samples $\left(\mathbf{x}_{\mathbf{1}}, \theta_{\mathbf{1}}\right),\left(\mathbf{x}_{\mathbf{2}}, \theta_{\mathbf{2}}\right) \ldots\left(\mathbf{x}_{\mathbf{n}}, \theta_{\mathbf{n}}\right)$ and belong to the domain of nonparametric statistics.

Given the knowledge of $N$ prototype patterns (vectors of dimentsion $\Sigma$ ) and their correct classification into $M$ classes, the $k \mathrm{NN}$ rule assigns an unclassified pattern to the class that is most heavily represented among its $k$ neighbors in the pattern space (under some appropriate metric). The first formulation of this rule was done by Fix and Hodges [12] in 1951 who established the consistency of the rule for sequences such that $k \rightarrow \infty$ and $k / N \rightarrow 0$. Of course, the probability of error $R$ of this rule must be at least as large as the Bayes probability of error $R^{*}$ which could be achieved with perfect knowledge of the probability density functions of each class. Cover and Hart [11] showed that the conditional risk for the 1-NN rule is bounded by $R \leq R^{*}\left(2-\frac{M}{M-1} R^{*}\right)$ where $\mathrm{M}$ is the number of different classes. This is close to $R \leq 2 R^{*}$ when $R^{*}$ is small, as usual in practical applications. For the $k$-NN rule, the risk is bounded by $\left(1+\frac{1}{k}\right) R^{*}[11]$.

\section{C.2 classification k-NN in RGB color space}

Implementing the $k$-NN rule with a brute-force method to classify $F$ patterns with $N$ prototypes requires $F \times N$ distance computations and $o(F \times N \times \log (N))$ comparisons, which is prohibitive in our case.. Different approaches have been devised to improve algorithm performances. Some authors [13], [14], [15], [16] reduce the number of prototypes to consider while trying not to affect the accuracy of the resulting classification. Jiang and Zhang [17] decomposed hierarchically the prototypes into disjoint subsets and then applied a powerful tree-search algorithm. Friedman [18] orders the training data along the axis with the maximum sparsity for each pattern. This restricts the computations to a band around the projection of the test data onto this axis. Finally, An approach that is better suited to our problem was first proposed by Warfield [19] who applied it to double echo spin echo MR image classification. In such an application, the number of possible patterns is smaller than the number of patterns to classify, so that it becomes efficient to pre-compute a lookup table for every possible pattern, then to classify patterns by accessing this lookup table.

The computation of this lookup table is essentially a $k$ distance transformation problem. Distance transformation are algorithms that compute for every pixel of an image the distance to the nearest pixel of a given object. The $k$-DT algorithm used here is better detailed in Cuisenaire [20]. Briefly, a $k$-Euclidean distance transformation is computed by using ordered propagation to scan the pattern space, starting from the prototype patterns, then to their neighbors, then to their neighbors' neighbors and so on, by order of increasing Euclidean distance. The strict respect of the increasing distance order is achieved by bucket sorting of the propagation queue, which insures that no unnecessary computations are performed.

\section{C.3 Choice of parameters}

The parameters of this method are the number $F$ of training samples required, the number $k$ of nearest training samples used by the $k$-NN rule and the size $2^{D . B}$ of the pattern space where $D$ is the number of dimensions, i.e 3 for the RGB space, and $B$ the number of bits used to quantify each color. Empirically, we found that a few hundreds training samples per class were sufficient to yield good results. To determine the optimal $k$ and $B$, we performed 10-fold cross-validation by randomly dividing our training data in 10 sub-sets, then using 9 of those to train the classifier and the remaining one to evaluate the resulting error rate $R$..

\section{Segmentation of interstitial fibrosis}

Manual and automatic estimates were performed in a randomly selected image, as follows: using Scion Image Beta 4.0.2 Win software (available at http://www.scioncorp.com), the different surfaces were manually dessigned and the correspondig surfaces in pixels calculated. Automatic algorithm was implemented in VTK 
software with a TCL script as interpreter. Two different expert operators performed the test during five replications and compared them with five automatic replications. The data were analyzed with Sygmastat software version 2.0.3 (SPSS). The intraoperator reproducibility was determined by calculating the coefficient of variation $(C V=S D / m)$. Intra and inter-operator variabilities in the manual segmentation of MT, CD and CI were assessed with a two-way ANOVA test, using replication and operators as factors, followed by Tukey's test as a post hoc test. In all tests, the differences were considered to be significant when $p<0.05$.

\section{RESUlts}

A subsection of a typical muscle image is illustrated in the upper part of Figure 1. In this case, the image subset $\left(59.4 \times 63.4 \mu \mathrm{m}^{2}\right)$ shows the MT surface colored in yellow, the $\mathrm{CD}$ in red and the CI reaction in pink. Note the high tissue deformation introduced by fibrosis.

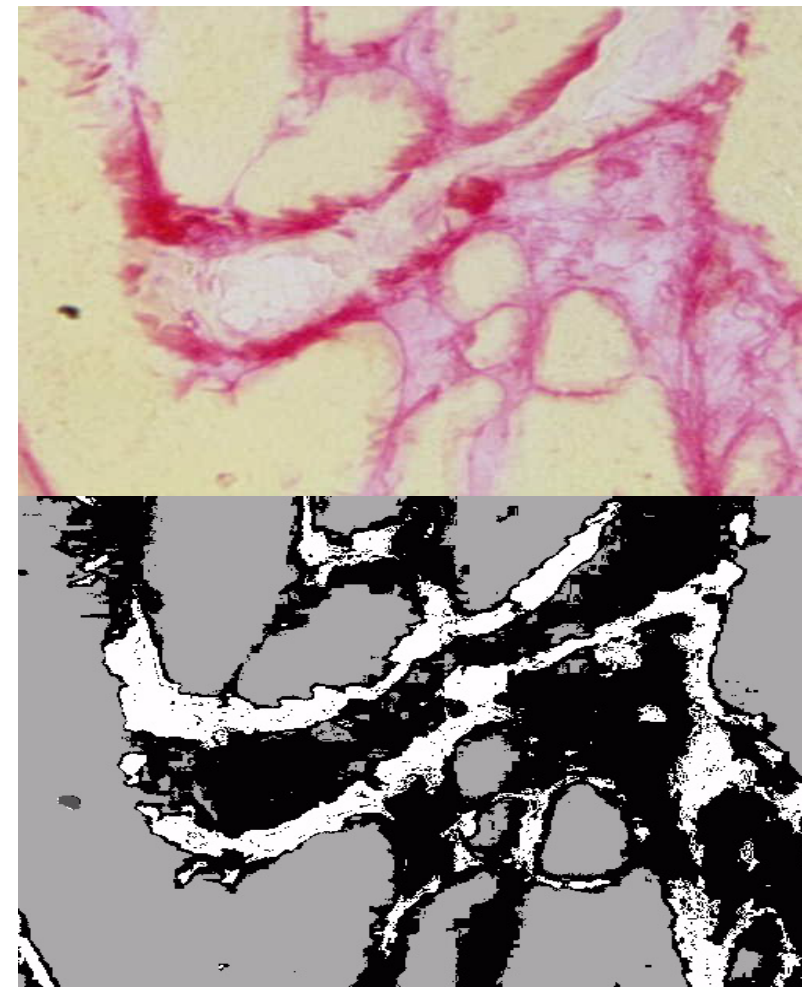

Fig. 1. Top figure shows an image subset $\left(59.4 \times 63.4 \mu \mathrm{m}^{2}\right)$ in which appeared the different features to segmentate. Bottom image corresponds to the segmented subset image. Four different colors define the classes defined on this image. Pixels colored in white correspond to the CD stained in red in upper image. Black pixels to the CI, colored in pink,

Four different classes were defined on this image: MT, CD, CI and artifact. Artifact class corresponds to staining failures and usually are presented as black spots. Segmentation, identification and classification of these features were achieved by using 162 (artifact), 297 (MT), 311 (CD), and 318 (CI) corrected classified pixels. In the bottom part, the resulted segmentated image is displayed, for a $k=11$. Pixels are colored in white, black and two different gray levels for the CD,CI, MT and artifact classes, respectively.

\section{A. Parameter selection}

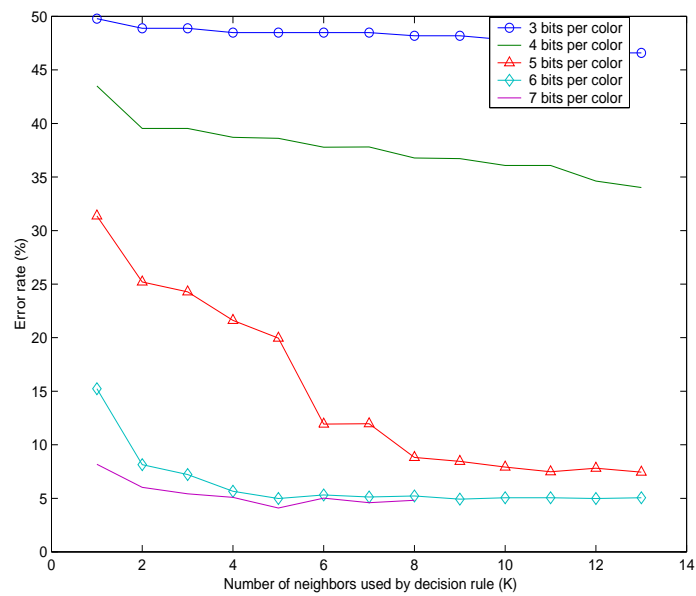

Fig. 2. Error percentage plotted against $k$ for different quantization steps.

The error rates $R$ for $1 \leq k \leq 13$ and $3 \leq B \leq 7$ are shown at Figure 2. Satisfying results are obtained as long as $k \geq 5$ and $B \geq 6$. For $k=5$ and $B=6$, the size of the $k$-DT space is $5 \times 8^{6}=1310720$, which requires a couple of seconds to compute the LUT. CPU time increases linearly with $k$ and is multiplied by 8 for each extra bit used to quantify the colors. Practically, we routinely use $k=11$ and $B=6$ or 7 .

\section{B. Manual-Automatic comparisons}

Manual measurements performed in the same image for five replication were statistically tested. Results are shown in table 1.

TABLE I

Manual-Manual COMParisons Were Performed USING the SAME image. Conventions: Inter: Interoperator Differences; Intra: Intraoperator differenCEs. Significant When $p \leq 0.05$, MARKED WITH ASTERISK.

\begin{tabular}{|c|c|c|}
\hline Tissue & Inter & Intra \\
\hline MT & $p=0.266$ & $p=0.072$ \\
\hline CD & $p=0.042^{*}$ & $p=0.849$ \\
\hline CI & $p=0.088$ & $p=0.587$ \\
\hline
\end{tabular}

Data show significant interoperator differences when considering the CD tissue. All other comparisons did not show significant differences.

TABLE II

MANUAL-Automatic COMPARISONS WERE PERFORMED USING THE same image. Same conventions as in table 1.

\begin{tabular}{|c|c|c|}
\hline Tissue & Inter & Intra \\
\hline MT & $p=0.561$ & $p=0.895$ \\
\hline CD & $p=0.183$ & $p=0.687$ \\
\hline CI & $p=0.706$ & $p=0.601$ \\
\hline
\end{tabular}


Table 2 shows statistical tests performed with the set of measurements obtained by the same operator (both automatic and manual). Data did not differ significantly.

\section{TABLE III}

COEFFICIENT OF VARIATION FOR THE TWO EXPERT OPERATORS AND THE AUTOMATIC METHOD DURING FIVE REPLICATIONS IN Percentage. Conventions: Op1: Operator 1; Op2; Operator 2; Auto: Automatic method.

\begin{tabular}{|c|c|c|c|}
\hline Tissue & Op1 & Op2 & Auto \\
\hline MCT & $1 \%$ & $1 \%$ & $1 \%$ \\
\hline PCBF & $11 \%$ & $16 \%$ & $11 \%$ \\
\hline CISLC & $22 \%$ & $6 \%$ & $7 \%$ \\
\hline
\end{tabular}

Finally, table 3 shows the CV for the two operators and the automatic method. Data show a larger dispersion for the two manual measurements.

\section{Discusion}

Compared with conventional methods, automatic approaches supplies additional new information and provides faster, more precise and reproducible quantifications. In the present study, we propose a novel image analysis automatic algorithm to segment and classify areas of interstitial fibrosis. The algorithm allows to quickly obtain accurate segmentations. Lookup table creation mean is about 12 seconds/image and image quantification about 4 seconds/image, depending on the number of selected prototype pixels and the $k$-dimensional problem i.e. the number of neighbors on which the decision is based. Also, as shown before, the interoperator variabilities of manual measurements were significantly different, while intraoperator values for MT were close to the sensibility statistical threshold. This results show an important interoperator variability that are much less important with the automatic method. Also, reproducibility is much reliable with the automatic method. There are two main factors that influence the reproducibility. First, the probability distributions of the different colors in the image can overlap each other. This correspond to the Bayesian risk and can be diminished by increasing color image contrasts. This implies being extremely careful at all steps towards well stained sections. Second, the noise than can be introduced by the operator at selecting the initial well classified pixels and that is directly related to the image quality. On the other hand, with this method, the microscope acquisition settings become less important since the algorithm classifies the different colors based on the correctly classified patterns that the observator supplies. Of course, any effort to increase the difference between colors can improve the algorithm performance, but an acceptable eye naked definition is sufficient to yield reproducible results, as shown in last section.

In conclusion, our method produces fast, robust, fully reproducible, accurate and realiable measurements of the dif- ferent tissues present in muscle sections stained with Sirius red. An objective and accurate quantification of the intersticial fibrosis reaction is fundamental to detect early structural modifications in pathologies such as the Duchenne dystrophy. This reasoning can be easily extended to all pathologies in which fibrosis occupy a determinant role and in which morphometry of this changes is usually difficult due to the large irregularities in color and shape introduced by this kind of reaction, as can be deduced from Figure 1.

\section{REFERENCES}

[1] H.J. Gundersen, T.F. Bendtsen, L. Korbo, N. Marcussen, A. Moller, K. Nielsen, J.R. Nyengaard, B. Pakkenberg, F.B. Sorensen, and A. Vesterby, "Some new, simple and efficient stereological methods and their use in pathological research and diagnosis," APMIS, vol. 96, pp. 379-394, 1988.

[2] J.R. Nyengaard, "Stereologic methods and their application in kidney research," JASN, vol. 10, pp. 1100-1129, 1999.

[3] A. Remuzzi, M. Mazerka, G.N. Gephardt, A.C. Novick, B.M. Brenner, and G. Remuzzi, "Three dimensional analysis of glomerular morphology in patients with subtotal nephrectomy," Kidney, vol. Int 48, pp. 155-162, 1995.

[4] E.R. Weibel, Stereological methods I: Practical methods for biological morphometry, London: Academic Press, 1989.

[5] M.J. Cullen and J.J Fluthrope, "Stages in fiber breakdown in duchenne muscular dystrophy," J. Neurol. Sci., vol. 24, pp. 179$186,1975$.

[6] F. Cornelio and I. Dones, "Muscle fiber degeneration and necrosis in muscular dystrophy and other muscle diseases: cytochemical and immunocytochemical data," Ann. Neurol., vol. 16, pp. 694-701, 1984.

[7] A.E.H. Emery, Duchenne muscular dystrophy, 2nd ed, Oxford University Press, 1993.

[8] A.T.M. Hageman, F.J.M. Gabreels, and K.D. Liem, "Congenital myotonic dystrophy: a report on 13 cases and a review of the literature," J. Neurol. Sci., vol. 115, pp. 95-104, 1993.

[9] W.R. Markesbery, R.C. Griggs, and B. Herr, "Distal myopathy: electron microscopic and histochemical studies," Neurol., vol. 27, pp. 727-735, 1977.

[10] L.A. Romano, M.D. Ferder, I.Y. Stella, F. Inserra, and L.F. Ferder, "High correlation in renal tissue between computed imageanalysis and classical morphometrical analysis," J. Histotechnol., vol. 19, pp. 121-123, 1996.

[11] T.M. Cover and P.E. Hart, "Nearest neighbor pattern classification," IEEE Trans. on Inf. Theo., vol. IT-13, pp. 21-27, 1967.

[12] E. Fix and J.L. Hodges, "Discriminatory analysis, nonparametric discrimination," Tech. Rep. Project 21-49-004, Rept. 4, Contract AF41(128)-131, USAF School of Aviation Medicine, Randolf Field, Texas, February 1951.

[13] P.E. Hart, "The condensed nearest neighbor rule," IEEE Trans. on Inf. Theory, vol. 14, pp. 515-516, 1968.

[14] G.W. Gates, "The reduced nearest neighbor rule," IEEE Trans. on Inf. Theory, vol. 18, pp. 431-433, 1972.

[15] I. Tomek, "Two modifications of cnn," IEEE Trans. Syst. Man Cybernet., vol. 6, pp. 769-772, 1976.

[16] S. Belkasim, M. Shridhar, and M. Ahmadi, "Pattern classification using an efficient knnr," Patt. Recognit., vol. 25, pp. 1269-1274, 1992.

[17] Q. Jiang and W. Zhang, "An improved method for finding nearest neighbors," Patt. Recognit. Lett., vol. 14, pp. 531-535, 1993.

[18] J. Fiedman, F. Baskett, and L.J. Shustek, "An algorithm for finding nearest neighbors," IEEE Trans. Comput., vol. 24, pp. 1000-1006, 1975.

[19] S. Warfield, "Fast k-nn classification for multichannel image data," Pattern Recog. Lett., vol. 17, pp. 713-721, 1996.

[20] O. Cuisenaire and B. Macq, "Fast k-nn classification with an optimal k-distance transformation algorithm," Proc. of the 10th European Signal Processing Conference, pp. 1365-1368, September 2000 . 